\title{
Estimación del potencial de captura de carbono en dos especies del género Polylepis, Recuay - Ancash.
}

Estimation of carbon capture potential in two species of the genus Polylepis, Recuay - Ancash.

$$
{ }^{1} \text { Eleuterio Ramírez Apolinario }{ }^{\mathrm{a}}{ }^{1} \text { Peláez Peláez Freddy }{ }^{\mathrm{b}}
$$

\section{RESUMEN}

El objetivo de esta investigación fue estimar el potencial de captura de carbono en especies de Polylepis incana y Polylepis serícea, que crecen en bosques nativos del caserío de Poccrac, distrito de Ticapampa, Ancash. Las ecuaciones alométricas para ambas especies, relacionando la biomasa (M) como variable dependiente con el diámetro de altura de pecho como variable independiente (DAP). La medida del DAP de los árboles de Polylepis talados, están dentro del rango que va desde $5 \mathrm{~cm}$ a $20 \mathrm{~cm}$. En el análisis de regresión realizado con el programa estadístico SPSS V 22.0 ,encontramos para la especie Polylepis incana un coeficiente de determinación de $\mathrm{r}^{2}=0.929$ y para la especie Polylepis sericea un $\mathrm{r}^{2}=0.94$, lo cual indica un alta relación entre las variables biomasa y DAP, mostrando buena bondad de ajuste para los parámetros que constituyen la ecuación alométrica de ambas especies. Los resultados obtenidos constituyen un importante aporte metodológico para las estimaciones de captura de carbono en zonas destinadas a la conservación y una herramienta para valorar la función de captura de $\mathrm{CO}_{2}$ en bosques andinos.

Palabras clave: biomasa, ecosistemas andinos, ecuaciones alométricas.

\begin{abstract}
The objective of this research was to estimate the potential for carbon capture in Polylepis incana and Polylepis sericea species, which grow in native forests of the village of Poccrac, district of Ticapampa, Ancash. He allometric equations for both species, relating the biomass (M) as a dependent variable with the breast height diameter as an independent variable (DAP). The DAP measurement of the cut Polylepis trees is within the range from $5 \mathrm{~cm}$ to $20 \mathrm{~cm}$. In the regression analysis performed with the statistical program SPSS V 22.0, we find for the Polylepis incana species a coefficient of determination of $\mathrm{r}^{2}=0.929$ and for the Polylepis sericea species $\mathrm{ar}^{2}=0.94$, which indicates a high relation between the variables biomass and DAP, showing good goodness of fit for the parameters that constitute the allometric equation of both species. The results obtained constitute an important methodological contribution for the estimates of carbon capture in areas destined for conservation and a tool to assess the $\mathrm{CO}_{2}$ capture function in Andean forests.
\end{abstract}

Keywords: biomass, Andean ecosystems, allometric equations. 


\section{INTRODUCCIÓN}

La actividad antrópica en la zona rural, el mal manejo y uso de los recursos naturales, las altas emisiones del dióxido de carbono, vienen ocasionando cambios en el planeta que son evidentes; el incremento de la temperatura del planeta, las concentraciones de los gases de efecto invernadero, las pérdidas de masas de nieve y hielo, entre otros, constituyen efectos palpables. A lo largo de la extensión de nuestro territorio nacional existen cadenas de montañas que sobrepasan altitudes $3000 \mathrm{msnm}$, que permiten el desarrollo de bosques en ecosistemas diversos y paisajísticamente extraordinarios los cuales son social y económicamente importantes . La mayoría de los ecosistemas podrían verse afectados por estas alteraciones que experimenta nuestro clima.(Bernier y Schoene, 2007)

La vegetación de los Andes Centrales está dominada por zonas agrícolas, pastizales y zonas arbustivas. Los bosques de Polylepis representan la vegetación natural de una gran parte de los Andes, a altitudes entre 3500 m. a 5 $200 \mathrm{~m}$. las especies del género ocupan una gran variedad de hábitats, desde el límite superior de los bosques de neblina hasta los volcanes áridos del Altiplano. Sin embargo, durante milenios las actividades y las necesidades humanas en los Andes han destruido estos bosques, restringiéndolos a hábitats especiales y modificando su composición florística y faunística.

El $\mathrm{CO}_{2}$ presente en la atmosfera se captura en los procesos metabólicos de las plantas a través de la fotosíntesis; en este proceso, el carbono atmosférico secuestrado se expresa en términos de biomasa constituido por follaje, ramas, raíces, troncos, flores y frutos (Rodríguez et al., 2006).. Siendo los arboles los que retienen en mayor cantidad este elemento por unidad de área en comparación con otros tipos de vegetación (IPCC, 2007).

En el Perú, los bosques andinos son remanentes de grandes extensiones de bosques de "quenual" (Polylepis spp.) y "colle" (Buddleia spp.), así también existen 19 especies del género Polylepis de las 28 que se tienen registradas y reportadas a nivel mundial, se habla de un centro de diversidad del género ya que concentra gran cantidad de especies. Un Decreto Supremo dictado en el año 2006, cuyo objetivo es identificar y priorizar elementos de la Diversidad Biológica que se encuentran en amenaza por lo que deben ser conservados y requieren atención prioritaria, brinda la Lista Roja de Especies de Flora y Fauna Amenazadas elaborada por la Unión Mundial para la Conservación, del cual se extrae que en la categoría de Peligro Crítico se encuentran las especies $P$. incana y $P$. racemosa, en Peligro comprenden P. microphylla, $P$. multijuga, P. subsericans y P. tomentella, en la categoría Vulnerable están las especies $P$. besseri, P. pepei, P.rugulosa, P. serícea, P. tarapacana y $P$. weberbaueri, por último en la categoría Casi Amenazado se encuentra P. pauta. Es decir 12 de las 19 especies presentes en el país requieren planes de conservación debido a las amenazas que atraviesan, sin embargo, el vacío de información existente sobre estas especies dificulta su conservación (Llerena et al. 2014; Mendoza y Cano, 2011).

En las Región Ancash, especialmente en la Cordillera Blanca, la vegetación mayormente se presenta en forma de pajonales y pequeños arbustos, siendo el género Polylepis, el elemento arbóreo dominante. 
Su tala, (a pesar de ser una especie protegida) está generando pérdida de la diversidad biológica, alteración del ciclo hidrológico y cambio micro meteorológico, afectando a la población local y a la vez acelerando la degradación del ecosistema. Su conservación representa una prioridad por su extraordinaria riqueza y endemismo, y porque varias de sus especies constituyentes están siendo severamente amenazados (Aubad et al., 2010)

Frente a esta problemática de debe buscar mecanismos de solución diferentes a los utilizados en la actualidad, que pasa solo por prohibir la tala de esta especie, actividad que no puede ser controlado en su totalidad debido a la extensión del área geográfica por donde se extienden los pequeños bosques de Polylepis $\mathrm{y}$ por la necesidad específica de cada población que se ubica en las cercanías de estos bosques.

Una alternativa viable según Ruignitz et al, (2009), sería realizar intervenciones productivas de servicios ambientales, que tengan como meta la estimación de captura de carbono en bosques de productores familiares o comunidades rurales, como una forma de generar beneficios financieros mediante el acceso al mercado de bonos de carbono. Restaurando las áreas degradadas por medio de plantaciones y/o regeneración natural, y por la extracción de madera. En ambos casos se pretende almacenar el carbono a través del crecimiento de árboles y, al extraer la madera, convertirla en productos durables. El carbono acumulado se mantendrá durante la vida útil del producto. Al extraer la madera, la regeneración actuará almacenando carbono por el crecimiento. Los sistemas forestales y agroforestales pueden capturar en sus diferentes almacenes de 80 a 350 toneladas de carbono por hectárea. (Camilo y Díaz 2010).
Avendaño et al., (2009), sostiene que: "la estimación de la biomasa en zonas que están dedicadas a la conservación requieren de la implementación de un método de medición con datos que se ajusten a la estructura particular de cada bosque sin la necesidad de generar tala de árboles". Ziani y Mencuccini, (2004); proponen métodos indirectos a partir de mediciones dasométricas precisas como por ejemplo el diámetro de altura de pecho( DAP), como una alternativa adecuada para la estimación de la biomasa en áreas donde existen restricciones para la tumba de árboles y se requieren resultados con bajo margen de error.

Entonces para esta investigación se ha utilizado modelos alométricos como metodología que permite obtener una estimación confiable y directa de la biomasa y por ende del carbono en los sistemas vegetales, porque tal estimación es individual y mediante la sumatoria se puede obtener una estimación global para cada sistema. (Etchevers et.al. 2002).

\section{MATERIALES Y MÉTODOS}

\section{Objeto de estudio}

Biomasa de plantas de Polylepis spp de un bosque interandino ubicado en la zona de Poccrac, Disrito de Ticapampa en Ancash. Especies: Polylepis incana y Poyilepis sericea.

\section{Selección de la muestra}

La selección de las plantas a talar se realizó empleando un muestreo no probabilístico de tipo intencional, es decir se seleccionó el tipo de planta de acuerdo a sus características dasometricas como: diámetro de altura de pecho (DAP), altura de copa, fuste recto y de fácil acceso. Las plantas taladas estuvieron en un rango de: $5 \mathrm{~cm} \leq \mathrm{DAP} \leq 20 \mathrm{~cm}$. 
Para ambas especies se realizó 11 talas. Éstas fueron hechas en el periodo que va de Mayo a Diciembre que es el periodo de carencia de lluvias en la zona sierra de Ancash.

Área de estudio

La recolección de datos se realizó en remanentes de un bosque natural, ubicado en las quebradas del Caserío de Poccrac, Distrito de TicapampaRecuay. Departamento de Ancash. Geográficamente situado entre las coordenadas: Latitud $09^{\circ} 4512811$ Sur.Longitud:77² 2614011 Oeste. Y una altitud de 3465msnm (Código Ubigeo: 021710-RENIEC).

\section{Métodos y técnicas}

En este trabajo se utilizó una metodología basada en la correlación entre el DAP y la biomasa, ya que según Solano et al. (2014), ésta constituye la variable que frecuentemente más se relaciona con la biomasa y es la más fácil de ser medida en campo, ya que la altura y otras variables son menos precisas de identificar y medir. Además, según el mismo autor, los modelos alometricos con variables logarítmicas y con exponentes cuadrados, son los que mejor grado de significancia tienen; es decir son los modelos más confiables y que mejor se ajustan para calcular la biomasa aérea de las especies forestales.

\section{Estimación de biomasa}

Para estimar la biomasa se utilizó el método indirecto, también llamado no destructivo, que consiste en cortar y pesar una cantidad definida de árboles, el peso (M) de estas plantas se relaciona con una variable dasométrica de fácil medición, que en nuestro caso es el diámetro de altura de pecho (DAP), luego se observa el comportamiento de la relación en un gráfico $(\mathrm{M}$ vs DAP). Mediante análisis de regresión se forma una ecuación que se denomina alométrica, que puede ser extrapolada a toda la población en estudio.

Ecuación alométrica

La relación $\mathrm{M}$ vs DAP, tiene comportamiento polinomial de la forma:

$\mathrm{M}=\mathrm{B}(\mathrm{DAP})^{\mathrm{A}}$ 1

Dónde: $\mathrm{M}=$ Biomasa aérea; $\mathrm{DAP}=$ Diámetro de altura de pecho $(1.30 \mathrm{~m}$ de altitud de la planta respecto al suelo); A y B son parámetros para estimar

La expresión (1) se linealiza aplicando logaritmo natural a ambos lados de la ecuación: $\mathrm{LNM}=\mathrm{LNB}+\mathrm{A} \mathrm{LN}(\mathrm{DAP})$ .2

Es decir la expresión (2), coincide con una ecuación lineal de la forma:

$$
\mathrm{Y}=\mathrm{a}+\mathrm{b} \mathrm{X}
$$

Donde: $\mathrm{Y}=\mathrm{LNM} ; \mathrm{X}=\mathrm{LN}(\mathrm{DAP}) ; \mathrm{b}=\mathrm{LNB}$ (intercepto de línea recta); $\mathrm{a}=\mathrm{A}$ (pendiente de línea recta); Las contantes: a, y b. (Son parámetros para estimar)

\section{Estimación de los parámetros de la ecuación alométrica}

\section{Método de mínimos cuadrados}

Para estimar los valores de los parámetros a y b de la recta que mejor se ajusta a los datos experimentales, se utilizó el método de mínimos cuadrados. El valor de éstos parámetros se determina de la siguiente manera:

$$
a=\frac{n \sum x y-\sum x \sum y}{n \sum x^{2}-\left(\sum x\right)^{2}} \quad b=\frac{\sum y-a \sum x}{n}
$$




\section{Bondad de ajuste}

El criterio más sencillo para determinar la bondad de ajuste para una recta de regresión lo da el coeficiente de correlación de Pearson:

$$
r=\frac{\sum(x-\bar{x})(y-\bar{y})}{\sqrt{\sum(x-\bar{x})^{2}} \sqrt{\sum\left(y-\bar{y}^{2}\right.}}
$$

\section{Coeficiente de determinación:}

El coeficiente de determinación expresa la cantidad de variación de la variable dependiente que es explicada por la variable independiente. Cuanto más próximo a uno es el valor de r2 mejor fue realizado el ajuste. Machado et al.(2002).

$$
r^{2}=\left[\frac{\sum(x-\bar{x})(y-\bar{y})}{\sqrt{\left(x-\bar{x}^{2}\right.} \sqrt{\sum\left(y-\bar{y}^{2}\right.}}\right]^{2}
$$

\section{Técnica de recolección y pesaje de muestras}

En la toma de datos se procedió a medir el DAP de la planta antes de talar, luego de la tala se separaron las partes: fuste, ramas y hojas y se pesaron separados. Luego del talado y el pesado en campo, se procedió a trasladar las plantas a un almacén para su secado respectivo.

El secado se realizó de manera natural y en sombra, para ello se monitoreo cada semana el avance de secado hasta que alcanzó un peso constante (que aproximadamente sucede a las 8 semanas), a partir de este valor se considera el peso en seco de la planta. Datos consignados en la tabla 1, para la especie Polylepis Incana y la tabla 2 para la especie Polylepis Sericea.

\section{RESULTADOS}

Tabla1. Datos del diámetro de altura de Pecho (DAP) y de la biomasa aérea de Polylepis Incana

\begin{tabular}{lccccccccccc}
$\mathrm{N}^{\circ}$ & 1 & 2 & 3 & 4 & 5 & 6 & 7 & 8 & 9 & 10 & 11 \\
\hline $\mathrm{DAP}(\mathrm{cm})$ & 11,2 & 10,5 & 16,0 & 17,0 & 18,0 & 9,1 & 6,5 & 7,9 & 12,0 & 13,5 & 6,0 \\
$\mathrm{M}(\mathrm{Kg})$ & 16,5 & 12,9 & 25,1 & 28,5 & 35,5 & 14,0 & 5,62 & 6,4 & 18,9 & 28,5 & 3,2
\end{tabular}

Tabla 2. Datos del diámetro de altura de pecho (DAP) y de la biomasa aérea de Polylepis Sericea

\begin{tabular}{lccrrrrrrrrr}
\hline $\mathrm{N}^{\circ}$ & 1 & 2 & 3 & 4 & 5 & 6 & 7 & 8 & 9 & 10 & 11 \\
\hline $\mathrm{DAP}(\mathrm{cm})$ & 17,5 & 12,0 & 13,0 & 10,5 & 18,0 & 9,3 & 6,3 & 8,1 & 12,6 & 14,0 & 5,8 \\
$\mathrm{M}(\mathrm{Kg})$ & 29,0 & 17,0 & 24,0 & 13,1 & 36,0 & 14,2 & 5,5 & 6,6 & 20,4 & 28,0 & 3,0 \\
\hline
\end{tabular}


Para elaborar los modelos alometricos para ambas especies, se procedió a organizar un grafico con la información de biomasa (M) y el diámetro de altura de pecho (DAP). En ella se observa un comportamiento de tipo polinomial de la forma $\mathrm{M}=\mathrm{B}$ (DAP) A, tal y como se observa en la Figura 1 para Polylepis incana y figura 2, para Polylepis serícea.

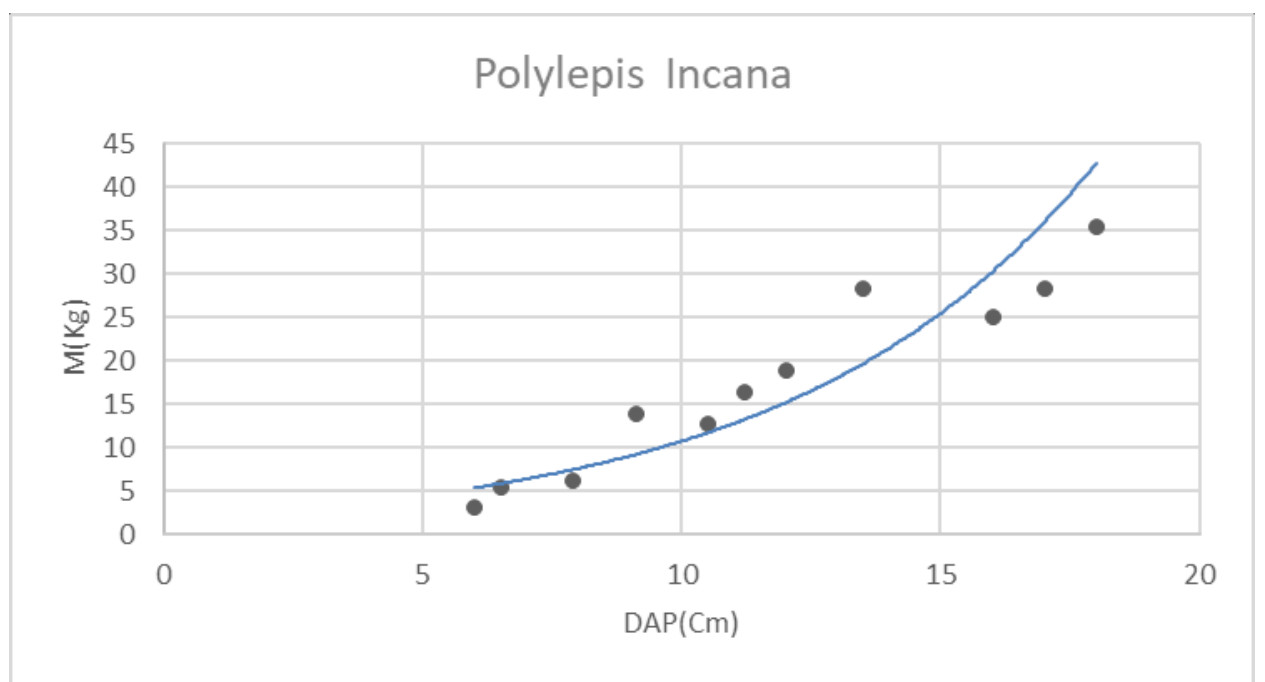

Figura 1. Comportamiento polinomial de la relación biomasa aérea y $D A P$, para Polylepis incana

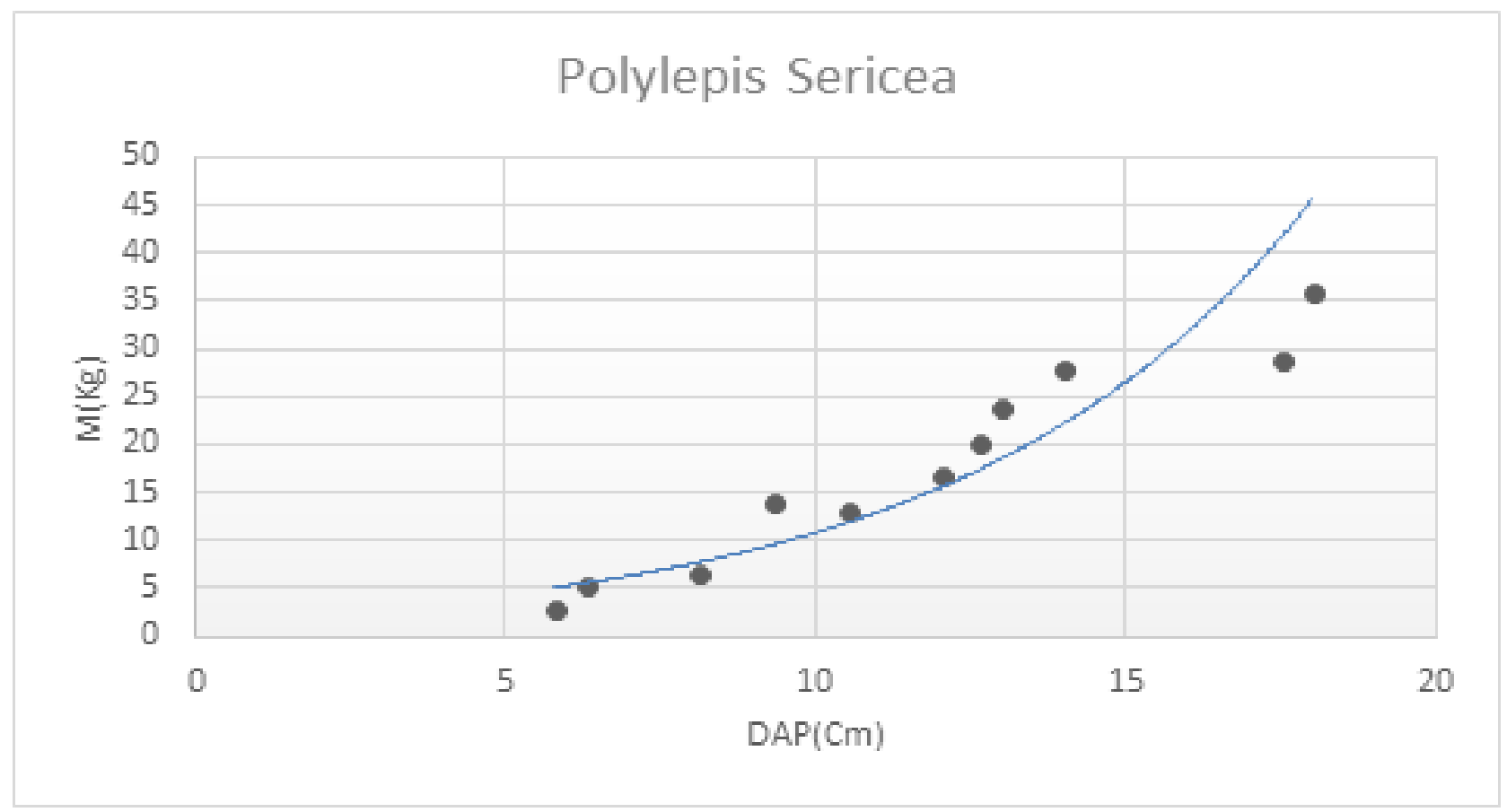

Figura 2. Comportamiento polinomial de la relación biomasa aérea y $D A P$, para Polylepis Sericea

La ecuación polinomial fue transformado a una forma lineal aplicando logaritmo natural a ambos lados de la ecuación, luego los parámetros A y B, fueron estimados con el método de mínimos cuadrados, resultando valores ubicados en la tabla 3 y en la tabla 4. 
Tabla 3. Datos obtenidos al linealizar la función polinomial, aplicando logaritmo natural a las variables, diámetro de altura de pecho (DAP) y biomasa aérea de la especie de Polylepis Incana.

\begin{tabular}{lccccccccccc}
$\mathrm{N}^{\circ}$ & 1 & 2 & 3 & 4 & 5 & 6 & 7 & 8 & 9 & 10 & 11 \\
\hline LNDAP (cm) & 2,416 & 2,351 & 2,773 & 2,833 & 2,890 & 2,208 & 1,872 & 2,067 & 2,485 & 2,603 & 1,792 \\
LNM (Kg) & 2,803 & 2,557 & 3,223 & 3,350 & 3,570 & 2,630 & 1,726 & 1,856 & 2,939 & 3,350 & 1,163
\end{tabular}

Tabla 4. Datos obtenidos al linealizar la función polinomial, aplicando logaritmo natural a las variables, diámetro de altura de pecho (DAP) y biomasa aérea de la especie de Polylepis Sericea

\begin{tabular}{lccccccccccc}
$\mathrm{N}^{\circ}$ & 1 & 2 & 3 & 4 & 5 & 6 & 7 & 8 & 9 & 10 & 11 \\
\hline LNDAP (cm) & 2,862 & 2,485 & 2,565 & 2,351 & 2,890 & 2,230 & 1,841 & 2,092 & 2,534 & 2,640 & 1,758 \\
LNM (Kg) & 3,367 & 2,833 & 3,178 & 2,573 & 3,584 & 2,653 & 1,716 & 1,887 & 3,015 & 3,332 & 1,099
\end{tabular}

Al relacionar el Ln de la biomasa y el Ln del DAP, en ambas especies, resulta una relación lineal positiva entre la biomasa y la variable DAP, se puede observar que el modelo funciona bien para las variables consideradas.

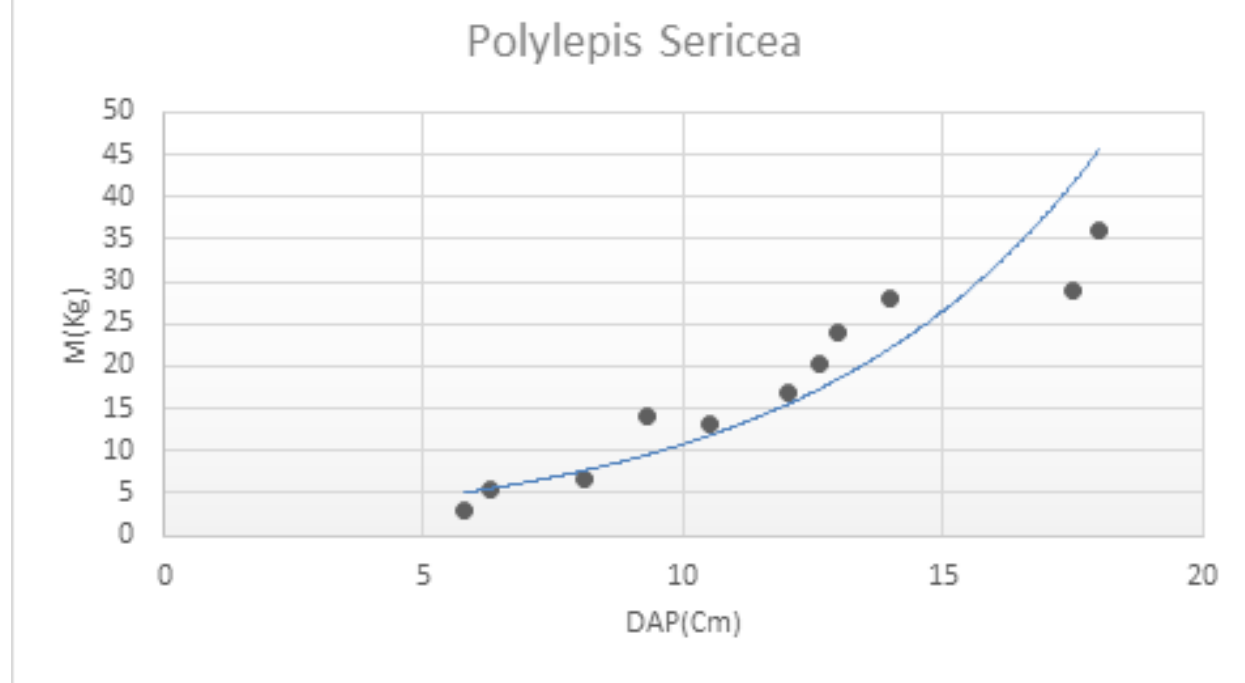

Figura 3. Comportamiento lineal de la relación biomasa aérea y DAP, para Polylepis Incana . 


\section{Polylepis Sericea}

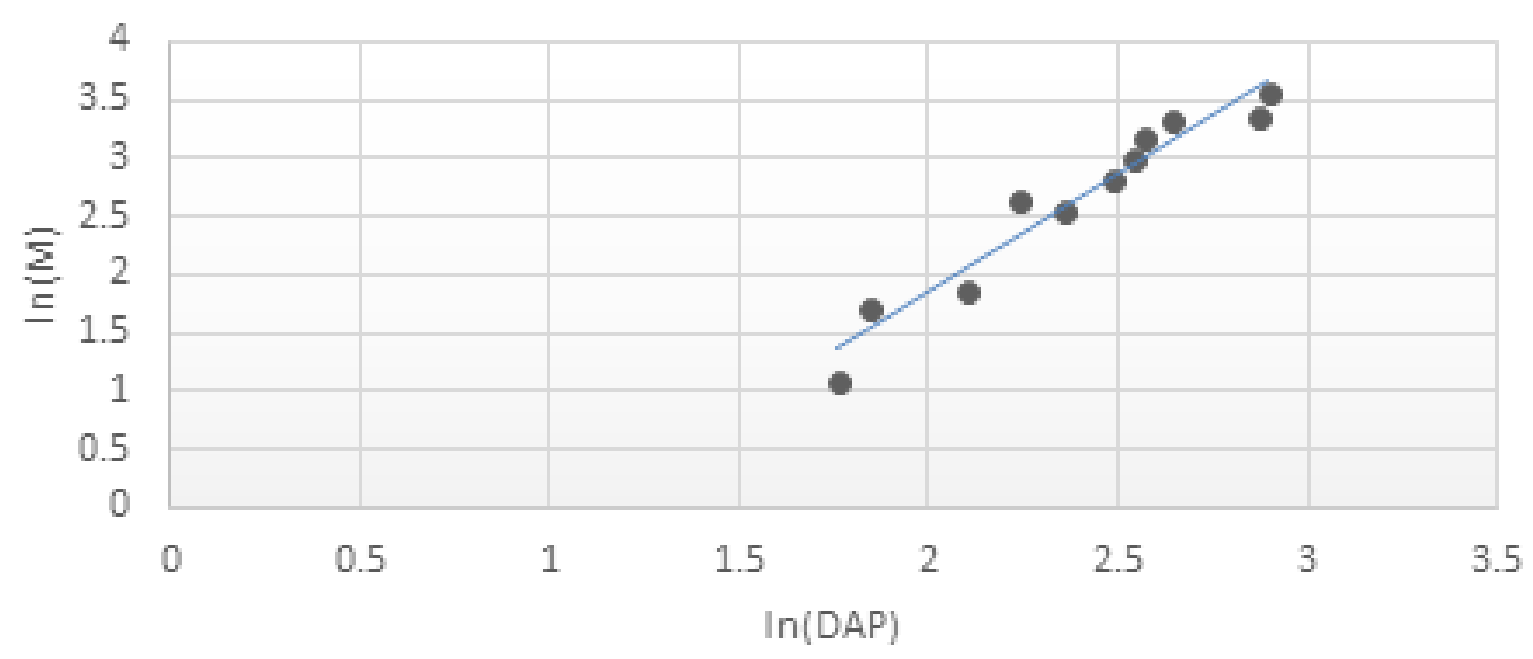

Figura 4. Comportamiento lineal de la relación biomasa aérea y DAP, para Polylepis Sericea.

Tabla 5. Resultado de análisis de regresión lineal con el programa estadístico SPSS V 22.0

Coeficientes

Nombre Constante LNDAP $\quad \mathrm{N} \quad \mathrm{r} \quad \mathrm{r}^{2} \quad \mathrm{r}^{2}$ corregido $\quad$ Error tipo residual

$\begin{array}{llllllll}\text { Polylepis incana } & -2,137 & 2,0078 & 11 & 0,968 & 0,936 & 0,929 & 0,043 \\ \text { Polylepis sericea } & -2.1757 & 2,0250 & 11 & 0,970 & 0,941 & 0,934 & 10,04\end{array}$

\section{DISCUSIÓN}

Para el análisis de la regresión lineal de las ecuaciones alométricas se utilizó el programa estadístico SPSS V 22.0; la cual arrojó como resultado valores de r2 0.929 para Polylepis incana y r2=0.934 para Polylepis sericea; esto indica que para ambas especies existe una fuerte correlación entre las variables estimadas. El 93.6\% de la variación de la variable biomasa de Polylepis incana se debe a la presencia de la variable DAP en el modelo . El 6.4\% se debe a otros factores intervinientes. Para el caso de Polylepis sericea, el 94.1\% de la variación de la variable biomasa se debe a la presencia de la variable DAP en el modelo. El 5.9\% se debe a otros factores intervinientes.
El valor alto del coeficiente de determinación (r2) indica una gran variabilidad en los datos y que el modelo para ambas especies es muy adecuado y significativo. El estudio está restringido para diámetros de fuste en un intervalo de, $5 \mathrm{~cm} \leq \mathrm{DAP} \leq 20 \mathrm{~cm}$, por lo tanto la validez de las ecuaciones se dan para este rango de medición, para la misma especie y que presenten características similares de crecimiento en la zona.

El DAP como variable independiente es un buen predictor de la biomasa aérea de las especies estudiadas, ya que las ecuaciones alométricas obtenidas dieron $\mathrm{r} 2$ cercanos a la unidad. Aunque varios estudios dicen encontrar una buena relación de la biomasa cuando se usan simultáneamente las variables diámetro y altura, 
en la mayoría de los casos con solo emplear el DAP también se logra una buena estimación, tal y como lo afirman Brown (2001) y Acosta et al. (2002). Las constantes de la ecuación alométrica para Polylepis Incana reportado por la siguiente investigación, es comparable a lo encontrado para la misma especie por Schlegel et al. (2001), quien establece que, los modelos que mejor estiman biomasa son los de tipo alomerico, de la forma $\mathrm{Y}=\mathrm{a} \mathrm{Xb}$ con transformación logarítmica que incluye variable de DAP. La validez de las ecuaciones alometricas generadas en este estudio debe restringirse al rango de variación de las dimensiones de DAP para un intervalo de $5 \mathrm{~cm} \leq \mathrm{DAP} \leq 20 \mathrm{~cm}$

\section{CONCLUSIONES}

La ecuación alométrica calculada para la estimación de la biomasa aérea es altamente confiable, ya que el valor del r2 para las dos ecuaciones es superior a 0.9 , lo cual muestra alta dependencia entre las variables analizadas en los gráficos de correlación, lo que quiere decir que puede ser usada para estimaciones de este componente restringidos solo para Polylepis Incana y Polylepis Sericea, debido a que la ecuación fue desarrollada bajo las características propias de estas especies. La información obtenida en el presente trabajo, podrá ser utilizada como información de entrada para modelización de escenarios de cambio climático y como linea base en futuros estudios de conservación de la especie.

\section{REFERENCIAS BIBLIOGRÁFICAS}

Avendaño, R., Galindo, A. And Angulo, A. 2009. Ecología y educación ambiental. Universidad autónoma de Sinaloa Culiacan. Mexico DF.

Acosta M. , Vargas H., Velasquez M. y Etchevers B. 2002. estimación de la biomasa aérea mediante el uso de relaciones alométricas en seis especies arbóreas en oaxaca, mexico. agrociencia 36. $752-736$

Aubad, J., Aragón, P. y Rodríguez, 2010. Human access and landscape structure effects on Andean forest bird richness, Acta Oecologica 36: 396-402

Brown, S. (2001). Estimating biomass and biomass change of tropical forest: a primer. Roma. FAO forestry paper, 134.

Bernier, P. y Schoene, D. 2007. La adaptación de los bosques y su ordenación al cambio climático.

Camilo, P.; y Diaz, T. 2010. Estimación del carbono contenido en la biomasa forestal aérea de dos bosques andinos en los Departamentos de Santander y Cundinamarca. Colombia. UDFJC.

Etchevers.J; Vargas J; M. Acosta; A. Velásquez .2002. Estimación de la biomasa, aérea mediante el uso de relaciones alometricas en seis especies arbóreas en OaxacaMéxico Agrociencia. Vol.36. №006

IPCC (Intergovernmental Panel on Climate Change). 2007. Climate Change (2014): The Physical Science Basis. Summary for Policymakers. Paris, Francia. s.e. p 2.

Llerena, P.;S.Yalle ;E. Silvestre. 2014. Los bosques y el cambio climático en el Pero: Situación y perspectivas. Organización de la Naciones Unidas para la alimentación y la agricultura. Lima-Perú.

Machado, A., Conceicao, B., Figueiredo,J 2002. Modelagem do volume individual para diferentes idades e regimes de desbaste em plantacoes de pinus oocarpa . Revista Ciencias Exactas e Naturais, Vol. ${ }^{\circ} 4$ Jul/dez. : 185-197. 
Mendoza, Wilfredo y Cano, Asunción. 2011. Diversidad del género Polylepis ( Rosaceae, Sanguisorbeae ) en los Andes peruanos. Revista Peruana de Biología. Vol. 18, no. 2, p. 197-200

Pearson, T. Walker, y Brown, S. 2005. Sourcebook for land use, land-use change and forestry projects. Winrock International and the BioCarbon Fund of the World Bank. 64 p.

Rodriguez, R. Jimenez, J. Aguirre, O. yTreviño, E. 2006. Estimación del carbono almacenado en un bosque de niebla en Tamaulipas, México. CIENCIA UANL 9: $179 \mathrm{p}$.

Rugnitz,M. T.; Chacón, M,L; Porro R. 2009. Guía para la determinación de carbono en pequeñas propiedades rurales $1^{\mathrm{a}}$ edición, Lima Perù: Centro Mundial Agroforestal (ICRAF)/ Consorcio Iniciativa Amazónica (IA)

Schlegel, B., Gayoso, J. y Guerra, J. 2001 . Medición de la capacidad de captura de carbono en bosques de Chile y promoción en el mercado mundial. Manual de procedimientos para inventarios .Universidad Austral de Chile. Valdivia, Chile.

Solano,D.; C.vega, V. H. Heras, K.Cueva. 2014 .Generación de modelos alometricos para determinar biomasa aérea a nivel de especies, mediante el método destructivo de baja intensidad para el estrato de bosqque seco p $1 \mathrm{u} \mathrm{v}$ i e s t a c i o n a 1 d e 1 Ecuador.CEDAMAZ. Ecuador

Zianis D., Mencuccini M., 2003. On simplifying allometric analyses of forest biomass. Forest Ecology and Management, 187:
311-332.

\section{CORRESPONDENCIA:}

Mag. Eleuterio Ramirez Apolinario e-mail:era_0262@hotmail.com 